\title{
Angioedema-Induced by Angiotensin Converting Enzyme Inhibitors
}

\section{Blum Arnon*}

Interdisciplinary Stem Cell Institute, University of Miami, Miami, Florida 33136, USA

Keywords: Angioedema; ACE inhibitors

\section{Introduction}

Angioedema is a known allergic reaction to many medications. Angiotensin Converting Enzyme Inhibitors (ACE-I) have been reported to cause such an adverse event, however, the mechanism of action is not clear enough. ACE-I induced angioedema is often misdiagnosed, although it could develop to a life-threatening situation. It is believed that it is caused by bradykinin induced activation of vascular bradykinin B2 receptors. Studies have reported an incidence of angioedema of 1 in 1000 during the first week of use, and 1 to 42,000 subsequently. Life threatening airway obstruction requiring intubation has been reported in up to $22 \%$ of angioedema cases caused by ACE inhibitors, with an overall mortality rate of $11 \%$.

\section{Case Report}

A 57 years old man was admitted to our department because of severe swelling of the tongue and lips with shortness of breath and difficulty in swallowing for one day - without fever, flushing, syncope, urticaria, or abdominal pain. Medical history included Chronic Ischemic Heart Disease (he had coronary bypass graft surgery 10 years ago and afterwards had coronary intervention with stent insertion into 4 coronary arteries), and Diabetes Mellitus type II for 10 years (without nephropathy, retinopathy, or neuropathy) and was treated with oral hypoglycemic medications. He did not experience any urticaria or angioedema in the past, no history of allergy or drug related allergy, had no family history of angioedema, and he was consistently on his medications in the last 10 years without any on/off periods. His medications include Lisinopril $5 \mathrm{mg}$ once a day, Atenolol $25 \mathrm{mg}$ once a day, Glucophage $850 \mathrm{mg}$ once a day, and Aspirin $100 \mathrm{mg}$ once a day.

Physical examination was normal except for the swelling of the tongue and shortness of breath.

\section{Laboratory tests}

Hemoglobin $11.3 \mathrm{~g} \%$, WBC $8300 / \mathrm{mm}^{3}$ without hyper-eosinophilia or hyper-basophilia, PLT $218,000 / \mathrm{mm}^{3}$. Electrolytes were normal as well as renal function tests and liver function tests. Chest $\mathrm{X}$ ray was normal, and the electrocardiogram demonstrated a normal sinus rhythm, normal axis of $60^{\circ}, \mathrm{PR}$ and QRS were normal without signs of hypertrophy or ischemia.

The Lisinopril (ACE inhibitor) was discontinued and systemic steroids were initiated with a good clinical response within 3 hours. No recurrence was reported during a follow up period of 12 months.

\section{Comments}

The incidence of ACE inhibitor-induced angioedema is estimated to be between $0.1 \%-2.2 \%$. The mechanism of ACE inhibitor-induced angioedema is not fully understood because angioedema develops only in a minority of patients taking ACE inhibitors (a class effect), and its effect is idiosyncratic, not related with duration of treatment and it is not dose-dependent. The pathophysiology of angioedema induced by ACE-I resembles that of hereditary angioedema, mediated by bradykinin, activating vascular bradykinin $\mathrm{B} 2$ receptors. ACE is an important mediator in bradykinin degradation, increasing the steady state of bradykinin level in the blood. Besides increased bradykinin level caused by ACE blockage, other possible mechanisms include a bradykinin degradation pathway, and a diminished activity of dipeptidyl peptidase IV (the substance P degradation pathway) [1].

A retrospective analysis of 64 consecutive patients that were presented with angioedema (while receiving ACE inhibitors) found that 26 patients switched to angiotensin receptor blockers, 14 to a calcium antagonist, and 14 to other antihypertensive drugs with a positive clinical response. Angioedema disappeared or was reduced drastically upon withdrawal of the ACE inhibitor in 46 patients (85\%). Only a small percentage of patients with ACE inhibitor-related angioedema continue with this symptom when switched to an angiotensin receptor blocker [2]. Manifestations of ACE-I induced angioedema included swelling of the lips, mouth, tongue and airways. Other manifestations included edema of the skin and subcutaneous fat, involvement of the viscera that may cause nausea, diarrhea, and abdominal pain. ACE inhibitors should not be prescribed to patients with a history of hereditary or acquired Angioneurotic Edema. Immediate discontinuation of the ACE inhibitor following an episode of angioedema is mandatory, since continuation increases the risk of recurrent angioedema with serious morbidity [3]. A case series in England found that angioedema occurred in a disproportionate number of black patients [3], consistent with studies from North America that have demonstrated a markedly increased risk in black Americans (relative risk of 4.5) compared with white subjects [4]. The increased risk in black patients may be related to racial differences in the kallikrein-kinin system and increased sensitivity to bradykinin [3]. No sex predominance has been noted except for patients with gastrointestinal involvement which occurred exclusively in women. Smoking, increasing age ( $>65$ years), history of drug rash and seasonal allergies are also associated with increased risk. However, there is no tight association between ACE inhibitor associated cough and angioedema [5]. A recent report described treatment of 8 patients with ACE-I induced angioedema with the bradykinin B2 receptor antagonist icatibant that has been approved for treating hereditary angioedema [6]. Icatibant is a synthetic decapeptide that is similar in structure to bradykinin but contains 5 nonproteinogenic amino acids that differ in its structure and function from bradykinin. This conformational change blocks the bradykinin B2 receptors in a competitive inhibition mechanism, and causes relief of the angioedema symptoms within a few hours. The treated patients in this clinical trial had a rapid significant clinical improvement within 4 hours without any need for any further intervention [6].

\section{Conclusion}

ACE-I have been associated with angioedema in a small subset

*Corresponding author: Arnon Blum, Interdisciplinary Stem Cell Institute University of Miami, Florida, USA 33136; E-mail: navablum@hotmail.com

Received May 05, 2012; Accepted June 12, 2012; Published June 22, 2012

Citation: Blum A (2012) Angioedema-Induced by Angiotensin Converting Enzyme Inhibitors. J Clin Case Rep 2:153. doi:10.4172/2165-7920.1000153

Copyright: (c) 2012 Blum A. This is an open-access article distributed under the terms of the Creative Commons Attribution License, which permits unrestricted use, distribution, and reproduction in any medium, provided the original author and source are credited. 
of treated patients. The angioedema commonly involves the face and oropharyngeal tissues and may result in life-threatening airway compromise. The mechanism could be related to the bradykinin system, and black subjects may be at a higher risk to develop this complication. A recent bradykinin B2 receptor blocker that is used to treat hereditary angioedema has been shown to have a clinical beneficial effect on angioedema induced by ACE-I, and it may offer a practical solution to this life threatening situation.

\section{References}

1. Agah R, Bandi V, Guntupalli KK (1997) Angioedema: the role of ACE inhibitors and factors associated with poor clinical outcome. Intensive Care Med 23: 793796
2. Cicardi M, Zingale LC, Bergamaschini L, Agostoni A (2004) Angioedema associated with angiotensin-converting enzyme inhibitor use. Arch Intern Med 164: 910-913.

3. Gibbs CR, Lip GYH, Beevers DG (1999) Angioedema due to ACE inhibitors: increased risk in patients of African origin. Br J Clin Pharmacol 48: 861-865.

4. Brown NJ, Ray WA, Snowden M, Griffin MR (1996) Black Americans have an increased rate of angiotensin converting enzyme inhibitor-associated angioedema. Clin Pharmacol Ther 60: 8-13.

5. Kampitak T (2008) Recurrent severe angioedema associated with imidapril and diclofenac. Allergol Int 57: 441-443.

6. Bas M, Greve J, Stelter K, Bier H, Stark T, et al. (2010) Therapeutic efficacy of icatibant in angioedema induced by angiotensin-converting enzyme inhibitors: a case series. Ann Emerg Med 56: 278-282. 